\title{
Path Dependence of Adsorption Behavior of Mixtures Containing Dipalmitoyl Phosphatidylcholine
}

\author{
R. H. NOTTER, ${ }^{(25)}$ SHERYL SMITH, R. D. TAUBOLD, AND J. N. FINKELSTEIN \\ Departments of Pediatrics, Division of Neonatology [R.H.N., R.D.T., J.N.F.] and of Chemical Engineering [R.H.N., \\ S.S.J, University of Rochester, Rochester, New York, USA
}

\section{Summary}

The adsorption of aqueous phospholipid dispersions containing dipalmitoyl phosphatidylcholine (DPPC) is investigated at 35$37^{\circ} \mathrm{C}$ as a function of dispersion preparation technique. Systems studied in terms of surface pressure-time $(\pi-t)$ adsorption behavior were pure DPPC, 9:1 DPPC:dipalmitoyl phosphatidylethanolamine, 7:3 DPPC:egg phosphatidylglycerol (PG), and lipids extracted from cow lung lavage. The results show that $\pi$-t characteristics can differ significantly depending on the technique by which the DPPC-containing mixtures are initially dispersed in $0.15 \mathrm{M} \mathrm{NaCl}$ solution. Examples of path dependence include the fact that DPPC, which will not adsorb at $\mathrm{T}=35^{\circ} \mathrm{C}$ when placed in powdered crystals on the subphase surface, exhibits measurable $\pi$-t changes after subphase dispersion by sonication or by mechanical vortexing. For 7:3 DPPC:PG, dispersion by sonication on ice or by mechanical vortexing gives faster adsorption than dispersion by sonication without temperature control. The effect of heating to $\mathrm{T}=45^{\circ} \mathrm{C}$, which is greater than the gel to liquid crystal transition temperature of DPPC $\left(\mathrm{T}_{c}=41^{\circ} \mathrm{C}\right)$, is found to be particularly detrimental to the adsorption of 7:3 DPPC:PG. Of the phospholipid mixtures studied, extracted cow lung lipids exhibited by far the greatest adsorption capability and also showed less path dependence than 7:3 DPPC:PG. Similarly, in terms of dispersion techniques investigated, sonication on ice tended to give the most rapid adsorption for a given phospholipid mixture.

\section{Speculation}

Treatment of neonatal Respiratory Distress Syndrome by exogenous surfactant replacement with well-defined mixtures of synthetic phospholipids is an attractive potential alternative to the use of multicomponent lung extracts. However, the existence of a variety of possible metastable solution states for phospholipid mixtures requires that adsorption properties be characterized for each mixture as a function of dispersion technique. It appears probable that synthetic phospholipid mixtures of identical composition and apparent bulk concentration might give variable therapeutic results for different dispersion methods.

One area of current clinical concern involves the treatment of the neonatal Respiratory Distress Syndrome (RDS) with some type of lung surfactant replacement therapy (c.f. 14, 16, 17). Almost all such therapy involves the starting point of phospholipid dispersions in an aqueous bulk phase before delivery to the patient. Dispersion may be accomplished by a variety of methods, but sonication or mechanical vortexing are most often the techniques used. These techniques are known to give rise to different phospholipid solution states (e.g., multilamellar liposomes from vortexing as opposed to unilamellar liposomes from sonication at the same subphase conditions of $\mathrm{pH}$, ionic composition, etc.) (3, $5)$. In addition to physical state differences in solution, sonication or vortexing of a phospholipid mixture of a specific chemical composition may result in an aqueous dispersion of varying composition due to differences in the ease of dispersion of mixture components. Consequently, a clear probability exists for the formation of variable bulk solution states in lung surfactant replacement mixtures containing a high $\%$ of phospholipids. These different solution states can exhibit significant surface property differences. This raises the possibility that the therapeutic efficacy of a specific surfactant replacement mixture will not only be dependent upon mixture composition, concentration, and delivery method (which are currently receiving considerable attention), but also upon the solution preparation procedure.

One surface property that may depend strongly on dispersion technique is adsorption. As a first step to characterizing the potential magnitude of such effects on lung surfactant replacement, we investigated the adsorption of phospholipid mixtures containing dipalmitoyl phosphatidylcholine (DPPC) for a variety of initial conditions. The solution preparation method was the variable in all cases investigated, with subphase ionic composition, total surfactant concentration, and subphase temperature held constant. Experiments were done for pure DPPC and for the mixed systems of 7:3 (molar ratio) DPPC:phosphatidylglycerol (PG) and 9:1 DPPC:dipalmitoyl phosphatidylethanolamine (DPPE). In addition, adsorption experiments were performed on mixed lipids extracted by chloroform and methanol from calf lung lavage. The results support the interpretation that DPPC adsorption from aqueous dispersion differs depending upon the solution preparation method.

\section{MATERIALS AND METHODS}

Synthetic phospholipids. 1,2 dipalmitoyl-L- $\alpha$-phosphatidylcholine and 1,2 dipalmitoyl-L- $\alpha$-phosphatidylethanolamine were purchased from the Sigma Chemical Company, St. Louis, MO. Purity of each compound was verified by a single spot on thin layer chromatography (TLC), and by the presence of $>99 \%$ palmitic acid chains by gas chromatography. The PG was egg-PG, also from Sigma, and gave a single broad spot on TLC. DPPC, DPPE, and egg-PG were stored in chloroform solution under nitrogen at $-20^{\circ} \mathrm{C}$, and were used without further purification.

It has been recently reported (19) that small amounts of impurities $(<1$ mole $\%)$ can affect phospholipid surface properties such as apparent equilibrium spreading pressure. Our TLC determinations should be more accurate than this $1 \%$ value for many possible impurities, and they showed no contamination present. Moreover, the major purpose of the present work is to compare the adsorption properties of a specific phospholipid mixture prepared by varying methods, and this type of comparison should be insensitive to small impurity effects.

Cow lung lipids. Cow lung lipids (CLL) were obtained by the following procedure. Lungs from freshly sacrificed calves were purchased with trachea and primary bronchi intact from Conti 
Meat Packing Company, Rochester, NY. The lungs were lavaged with 2-3 liters of cold $0.15 \mathrm{M} \mathrm{NaCl}$ given in 5-6 divided doses. The resultant lavaged material was centrifuged at $300 \times g$ for 10 min to remove cellular debris. The supernatant was then centrifuged at $12,000 \times g$ for $30 \mathrm{~min}$; the pellet was resuspended, and the lipids extracted by the procedure of Bligh and Dyer (1). The phospholipids were analyzed by TLC in a solvent system of chloroform, methanol, 2-propanol, triethylamine and water in volume proportions of 30:9:25:25:7, respectively (20). The composition of the CLL was found as 95 weight\% phospholipid with a mole distribution of $79 \%$ phosphatidylcholines, 3.2\% phosphatidylethanolamine, 4.8\% phosphatidylglycerol (PG), 3.9\% phosphatidylinositol, $1.9 \%$ sphingomyelin, $7.2 \%$ other phospholipids. The fatty acid composition of the phosphatidylcholine fraction was found by gas chromatography to be $12: 0,2.5 \% ; 14: 0,5.6 \%$; $16: 0,72.5 \% ; 16: 1,5.9 \% ; 18: 0,1.9 \% ; 18: 1,9.0 \%$; other, $2.6 \%$. The $\%$ of cholesterol and cholesterol esters in CLL was $<5$ weight $\%$. Protein determination by a modification of the method of Lowry et al. (9) showed a trace of protein of the order of $0.5-0.75$ weight $\%$.

Mixture preparation. In all experiments, $5 \mathrm{mg}$ of surface active material (DPPC, 9:1 DPPC:DPPE, 7:3 DPPC:PG, CLL or 5:5 DPPC:CLL) were initially dispersed in $10 \mathrm{ml}$ of $0.15 \mathrm{M} \mathrm{NaCl}$ either by sonication for $1 \mathrm{~min}$ at room temperature or on ice, or by mechanical vortexing for $1 \mathrm{hr}$ at room temperature. These dispersions were accomplished with or without any additional heating to $45^{\circ} \mathrm{C}$, and with or without any aging, before being used in an adsorption experiment. The additional variables of heating and aging were chosen for several reasons. Heating the mixtures to a temperature higher than the gel to liquid crystal transition temperature $T_{c}$ of DPPC $\left(T_{c}=40-41^{\circ} \mathrm{C}\right)$, with subsequent cooling to room temperature, was intended to allow the phospholipids to assume any preferred solution state that they might not be able to achieve spontaneously at lower temperature. The aging variable was also to allow for any equilibration effects.

From this general framework of variables, specific dispersion preparation methods were used and identified as follows: $\mathbf{S}=$ sonicate for $1 \mathrm{~min}$ at room temperature; $S_{i}=$ sonicate for $1 \mathrm{~min}$ in an ice bath; $\mathrm{V}=$ vortex for a total time of $1 \mathrm{~h}$ without temperature control; $\mathrm{A}-1=$ age $1 \mathrm{~h} ; \mathrm{NA}=$ no age (i.e., use immediately); $\mathrm{H}$ $=$ heat given dispersion to $\mathrm{T}=45^{\circ} \mathrm{C}$ for one-half $\mathrm{h}$ for vortexed dispersions or $1 \mathrm{~h}$ for sonicated dispersions; and $\mathrm{NH}=$ no heating. For example, a mixture denoted by $\mathrm{S} / \mathrm{NH} / \mathrm{NA}$ was prepared by sonication for $1 \mathrm{~min}$ without temperature control, and then was used at once in an adsorption experiment without additional heating or aging.

Adsorption experiments. The adsorption experiments were conceptually simple and were similar in method to those used by King and Clements (7). Five mg of phospholipid were dispersed in $10 \mathrm{ml}$ of $0.15 \mathrm{M} \mathrm{NaCl}$ by a given method (22), and at time zero were added to an additional $70 \mathrm{ml}$ of $0.15 \mathrm{M} \mathrm{NaCl}$, which was being stirred in a teflon evaporating dish. The teflon dish used was $1.5 \mathrm{~cm}$ deep and had a surface area of $81.7 \mathrm{~cm}^{2}$. The experiments were done in an oven maintained with an air temperature of $37-40^{\circ} \mathrm{C}$. The initial subphase $(70 \mathrm{ml})$ was at $35-37^{\circ} \mathrm{C}$, but fell slightly upon addition of the $10 \mathrm{ml}$ phospholipid dispersion. This procedure was adopted so that subphase temperatures would consistently be slightly on the low side of $37^{\circ} \mathrm{C}$, to ensure that the $\mathrm{T}_{c}=41^{\circ} \mathrm{C}$ of DPPC was never exceeded during the adsorption process.

The $0.15 \mathrm{M} \mathrm{NaCl}$ subphase was stirred by a teflon stirring bar $(10 \mathrm{~mm} \times 3 \mathrm{~mm})$ by means of a magnetic stirrer. The rate of stirring was approximately constant in all experiments at a value greater than $100 \mathrm{rpm}$. This stirring rate was high enough so that discernable rippling effects could be seen at the subphase surface. Diffusion resistances in similar systems have been shown to be negligible compared to adsorption resistance (7). Subphase stirring was begun before $10 \mathrm{ml}$ of a given phospholipid dispersion was added, so that adsorption resistance would be the controlling effect even at small experimental times.

\section{RESULTS AND DISCUSSION}

The experimental results for pure DPPC are reported in Table 1 and show that this phospholipid does exhibit measurable adsorption at $\mathrm{T}<\mathrm{T}_{\mathrm{c}}$ after dispersal in an aqueous subphase by a variety of techniques. By contrast, DPPC exhibits negligible surface tension lowering when it is placed on a water surface in powdered crystal form below $T_{c}$, as found by Villalonga (21) and Phillips and Hauser (15), and as verified here in Table 1, case 2. The adsorption of DPPC is thus path dependent, and this correlates with the concept that, from a thermodynamic viewpoint, phosphatidylcholines below $T_{c}$ can exist in metastable onequilibrium states $(4,6,15)$.

Aside from the clear path dependence found for DPPC dispersions (case 2 versus cases 3-10 in Table 1), it is difficult to assign

Table 2. Adsorption of 9:1 (molar ratio) DPPC:DPPE for various solution preparation techniques

\begin{tabular}{lcccr}
\hline $\begin{array}{c}\text { Dispersal } \\
\text { method }^{1}\end{array}$ & $\begin{array}{c}\text { Surface pressure } \pi \\
\text { at } 20 \mathrm{~min}^{2}\end{array}$ & $\begin{array}{c}\pi \text { at } \\
60 \mathrm{~min}\end{array}$ & $\begin{array}{c}\pi \text { at } \\
120 \mathrm{~min}\end{array}$ & $\begin{array}{c}\pi \text { at } \\
180 \mathrm{~min}\end{array}$ \\
\hline 1. $\mathrm{S} / \mathrm{NH} / \mathrm{NA}$ & 3.1 dynes/cm & 6.4 & 8.8 & $10.5(n=4)$ \\
2. $\mathrm{V} / \mathrm{NH} / \mathrm{Na}$ & 2.3 & 3.3 & 4.6 & $5.4(n=3)$ \\
3. $\mathrm{S} / \mathrm{NH} / \mathrm{NA}$ & 0.8 & 3.3 & 5.7 & $7.0(n=3)$ \\
\hline
\end{tabular}

${ }^{1}$ See "Materials and Methods" for specific notation for preparation tchnique; apparent bulk phospholipid concentration was $62.5 \mu \mathrm{g} / \mathrm{ml}$.

${ }^{2}$ Surface pressure results are averages of several experiments $(n$ in parentheses).

Table 1. Adsorption of DPPC for various solution preparation techniques

\begin{tabular}{|c|c|c|c|c|}
\hline Dispersal method ${ }^{\mathrm{I}}$ & Surface pressure $\pi$ at $20 \mathrm{~min}^{2}$ & $\pi$ at $60 \mathrm{~min}$ & $\pi$ at $120 \mathrm{~min}$ & $\pi$ at $180 \mathrm{~min}$ \\
\hline $\begin{array}{l}\text { 1. No added DPPC (clean sur- } \\
\text { face) }\end{array}$ & 0 dynes/cm & 0 & 0 & 0 \\
\hline $\begin{array}{l}\text { 2. Powdered DPPC dropped on } \\
\text { surface }\end{array}$ & 0 & 0 & 0 & $0 \quad(n=4)$ \\
\hline 3. $\mathrm{S}_{\mathrm{i}} / \mathrm{NH} / \mathrm{NA}$ & 1.0 & 3.6 & 6.1 & $7.6(n=4)$ \\
\hline 4. S/NH/NA & 0.9 & 3.5 & 6.1 & $7.9(n=4)$ \\
\hline 5. S/H/NA & 0.7 & 1.1 & 1.6 & $2.3(n=3)$ \\
\hline 6. S/NH/A-1 & 0.5 & 2.1 & 4.4 & $6.1(n=3)$ \\
\hline 7. $\mathrm{S}_{\mathrm{i}} / \mathrm{H} / \mathrm{NA}$ & 0.6 & 1.4 & 2.7 & $4.1(n=2)$ \\
\hline 8. $\mathrm{S}_{\mathrm{i}} / \mathrm{NH} / \mathrm{A}-\mathrm{I}$ & 0.9 & 3.5 & 6.2 & $7.8(n=5)$ \\
\hline 9. $\mathrm{V} / \mathrm{NH} / \mathrm{NA}$ & 0.3 & 0.8 & 1.2 & $1.6(n=2)$ \\
\hline 10. $\mathrm{V} / \mathrm{H} / \mathrm{NA}$ & 0.7 & 2.0 & 2.8 & $3.8(n=3)$ \\
\hline
\end{tabular}

\footnotetext{
${ }^{1}$ See text under "Materials and Methods" for designation of specific preparation method; apparent DPPC bulk concentration was uniform for all experiments at $62.5 \mu \mathrm{g} / \mathrm{ml}$ (see reference 22).

${ }^{2}$ Surface pressure results are averages of several experiments (in parentheses).
} 
Table 3. Adsorption of 7:3 (molar ratio) DPPC:egg-PG for various solution preparation techniques

\begin{tabular}{lccccc}
\hline $\begin{array}{c}\text { Dispersal } \\
\text { method }^{1}\end{array}$ & $\begin{array}{c}\text { Surface pressure } \pi \\
\text { at } 20 \mathrm{~min}^{2}\end{array}$ & $\begin{array}{c}\pi \text { at } \\
60 \mathrm{~min}\end{array}$ & $\begin{array}{c}\pi \text { at } \\
120 \mathrm{~min}\end{array}$ & $\begin{array}{c}\pi \text { at } \\
180 \mathrm{~min}\end{array}$ \\
\hline 1. V/NH/NA & 12 & 17 & 22 & 27 & $(n=11)$ \\
2. V/H/NA & 1.2 & 2.2 & 8 & 14 & $(n=6)$ \\
3. $\mathrm{S}_{\mathrm{i}} / \mathrm{NH} / \mathrm{NA}$ & 15 & 23 & 32 & 37 & $(n=8)$ \\
4. $\mathrm{S}_{\mathrm{i}} / \mathrm{H} / \mathrm{NA}$ & 5 & 14 & 23 & 26 & $(n=3)$ \\
5. $\mathrm{S} / \mathrm{NH} / \mathrm{A}-1$ & 9 & 16 & 22 & 25 & $(n=4)$ \\
6. S/NH/NA & 5 & 12 & 17 & 21 & $(n=3)$ \\
7. S/NH/A-1 & 5 & 14 & 20 & 23 & $(n=2)$ \\
8. S/H/NA & 3 & 10 & 15 & 18 & $(n=2)$ \\
\hline
\end{tabular}

${ }^{1}$ See "Materials and Methods" for preparation technique notation; bulk phospholipid concentration was $62.5 \mu \mathrm{g} / \mathrm{ml}$.

${ }^{2}$ Surface pressure results are averages of several experiments ( $n$ in parentheses).

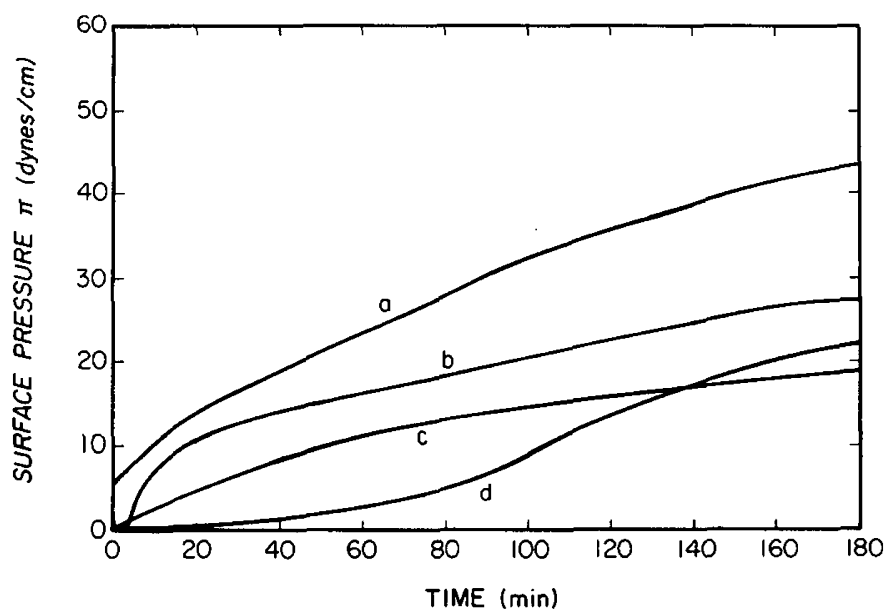

Fig. 1. Surface pressure $v s$. time adsorption behavior of 7:3 DPPC:PG dispersions. Curves shown are representative experiments chosen from the cases in Table 3. (a) Case of Si/NH/NA; (b) Case of V/NH/NA; (c) Case of $\mathrm{S} / \mathrm{NH} / \mathrm{NA}$; and (d) Case of $\mathrm{V} / \mathrm{H} / \mathrm{NA}$.

specific mechanistic interpretations to the $\pi$-time adsorption variations shown in Table 1. As noted in "Materials and Methods," at least some of the adsorption differences among cases 3-10 probably result from the varied effectiveness of a given method in dispersing DPPC in water, rather than from a direct effect of different physical states in solution. Also, because the overall magnitude of surface tension lowering achieved by DPPC in Table 1 is relatively small for any dispersion method, the variations found in $\pi$-t adsorption characteristics between different dispersion techniques were often of the same order as variations found between different experiments for the same dispersion technique. For example, the results for cases $3,4,6$ and 8 on Table 1 are felt to be essentially equivalent.

In spite of interpretational difficulties, the results of Table 1 clearly indicate that DPPC is able to exert higher surface pressures when dispersed in the subphase than is the case when it is placed on the surface in powdered form, and the adsorption of DPPC at $\mathrm{T}<\mathrm{T}_{\mathrm{c}}$ is consequently path dependent.

The same general conclusions reached for DPPC dispersions can be applied to the 9:1 (molar ratio) DPPC:DPPE results given in Table 2. The $\mathrm{T}_{\mathrm{c}}$ of DPPE has been reported as $59^{\circ} \mathrm{C}$ based on baseline departure by Blume and Ackerman (2) in differential scanning calorimetry studies, and as $63^{\circ} \mathrm{C}$ from the spin label determinations of Shimshick and McConnell (18). Interestingly, the differential scanning calorimetry studies (2) report an apparent decrease in $\mathrm{T}_{\mathrm{c}}$ to about $36^{\circ} \mathrm{C}$ in 9:1 DPPC:DPPE mixtures from the $40-41^{\circ} \mathrm{C} \mathrm{T}_{c}$ of pure DPPC. However, the spin label studies
(18) do not show such a decrease and give the $T_{c}$ of 9:1 DPPC:DPPE as about $41^{\circ} \mathrm{C}$. Thus, the 9:1 DPPC:DPPE system is probably slightly below its $T_{c}$ for the subphase temperatures of $35-37^{\circ} \mathrm{C}$ investigated here, and this is reflected in the relatively low surface pressures generated by the adsorption of this system. The $\pi$-t results for the 9:1 DPPC:DPPE system in Table 2 show a dependence on dispersion method, although the magnitude of the effect is small.

With the demonstration of path dependent adsorption for DPPC and 9:1 DPPC:DPPE dispersions, attention is now turned to three specific phospholipid mixtures of potential clinical importance. Table 3 reports results for the adsorption of a 7:3 DPPC:PG mixture as a function of dispersion technique. This particular mixture has current clinical relevance for the treatment of neonatal RDS as used by Morley and co-workers $(11,12)$. The adsorption behavior of 7:3 DPPC:PG shows surface tension lowering differences of large magnitude depending on dispersion path. This is not only true in terms of the final surface tension lowering achieved, but also in terms of the qualitative shape of the surface pressure-time $(\pi-t)$ curves, as shown in Figure 1. In particular, for the cases of a 7:3 DPPC:PG mixture either sonicated on ice or vortexed, and then used immediately with no heating (i.e., $\mathrm{V} / \mathrm{NH} / \mathrm{NA}, \mathrm{S}_{\mathrm{i}} / \mathrm{NH} / \mathrm{NA}$ under "Materials and Methods"), there is a rapid initial adsorption phase following subphase dispersal. After this initial rapid phase, there is a slower approach to final average surface pressures of the order of 27 dynes $/ \mathrm{cm}$ for vortexed mixtures and 37 dynes/cm for ice sonicated mixtures. Ice-sonicated 7:3 DPPC:PG mixtures displayed a more rapid initial adsorption phase than vortexed mixtures, as shown by curves $a$ and $b$ on Figure 1. A variable time lag before the onset of the rapid adsorption phase was also apparent for the case of vortexed mixtures.

The relatively good adsorption of 7:3 DPPC:PG mixtures dispersed by ice-sonication or by vortexing is in marked contrast to that observed for several other methods of bulk phase dispersion for the same system. For example, if dispersion of 7:3 DPPC:PG is accomplished by sonication or vortexing, but with heating to $45^{\circ} \mathrm{C}\left(\right.$ e.g., $\mathrm{V}_{\mathrm{i}} / \mathrm{H} / \mathrm{NA}$ or $\left.\mathrm{S}_{\mathrm{i}} / \mathrm{H} / \mathrm{NA}\right)$, the facility of adsorption is markedly reduced. The entire character of the $\pi$-time isotherm is changed to one of gradual adsorption with no initial rapid phase (curve $d$ on Fig. 1). This effect is more pronounced for vortexed mixtures (case 2 versus case 1 in Table 3) than for sonicated mixtures (case 4 versus case 3 in Table 3 ). However, heating to $T$ $>T_{c}$ is clearly detrimental to the adsorption facility of both vortexed and sonicated mixtures of 7:3 DPPC:PG.

Heating during the dispersion process might affect phospholipid adsorption for several reasons. For example, heating might cause a chemical change (e.g., hydrolysis) in the mixture. Heating could also give mixture components enough energy and mobilty to achieve a different bulk physical state, or it could affect the differential dispersion of mixture components. In terms of these potential mechanisms, chemical hydrolysis seem unlikely to be of major importance for the results of Table 3, because it can be argued that any hydrolysis products (e.g., free fatty acids or lysophosphatidylcholine) might increase rather than decrease adsorption. Detailed future experiments are required to characterize fully the mechanisms behind the detrimental effect of heating on $7: 3$ DPPC:PG mixtures.

The results of Table 3 imply that heating effects on this system can be quite subtle in some instances. Specifically, several experiments were done where mixture preparation procedure differed only in that sonication was done without temperature control or on ice (i.e., case 6, S/NH/NA and case $3, \mathrm{~S}_{\mathrm{i}} / \mathrm{NH} / \mathrm{NA}$ in Table 3 ). As seen from a comparison of these cases, the adsorption facility of 7:3 DPPC:PG is decreased if sonication is not done in an ice bath. One explanation for this effect is that uncontrolled heating during the sonication process was sufficient to harm adsorption capability. Some heating clearly did occur in our experiments of sonication without ice, because the phospholipid-water mixtures became warm to the touch even for the $1 \mathrm{~min}$, low power ( $25 \mathrm{watt}$ ) 
sonications being used. The results found for 7:3 DPPC:PG mixtures in Table 3 suggest that heating effects must be minimized to obtain maximal adsorption facility for this system. It is reasonable that this kind of effect will also prove important for other synthetic phospholipid mixtures besides those tested here.

Results for the adsorption of mixed lipids extracted from calf lung (see "Materials and Methods") are tabulated in Table 4. The most striking features of the CLL adsorption studies are the extremely rapid nature of the adsorption process, and the large surface pressure magnitudes $(46-47$ dynes $/ \mathrm{cm}$ ) achieved. These surface pressures are in the range of equilibrium spreading pressure found for many phosphatidylcholines above $T_{c}(15)$. Moreover, the adsorption facility of this CLL system is less path dependent than for 7:3 DPPC:PG mixtures, although the initial phase of CLL adsorption was again found to be faster after sonication on ice than after vortexing. For the case of ice sonication of CLL in Table 4, the process was found to occur with a rapid initial phase that took place on the order of sec. The initial adsorption was typically so rapid that it was difficult to measure its precise $\pi$-time dependence accurately on our chart recorder. Thus, although $\pi$ values for ice sonication are given at $1 \mathrm{~min}$ in Table 4 , the great majority of the $1 \mathrm{~min}$ value was generated in the first few sec after subphase dispersal. Dispersal of CLL by vortexing also gave rapid adsorption, but it was found to be slower in the initial phase than was the case following sonication in ice (cases 1 and 2 in Table 4 compared to cases 3 and 4). In addition, the vortexed CLL dispersions exhibited a point of inflection in the $\pi$-t curve during the initial rapid phase (not shown explicitly in Table 4). Thus, there were qualitative as well a quantitative differences in $\pi$ - $t$ behavior found for vortexed and sonicated CLL disperions (23). However, for both ice sonicated and vortexed CLL mixtures, adsorption was more rapid and caused more surface tension lowering than found for 7:3 DPPC:PG. We feel that the extremely rapid adsorption characteristics of extracted lung lipids may help to explain why mixtures composed of natural lung surfactant have thus far proven superior to artificial mixtures in animal model studies of exogenous replacement therapy for $\operatorname{RDS}(14,16,17)$.

The adsorption of CLL after dispersal by vortexing or by sonication on ice was not detrimentally affected by heating, as shown by a comparison of cases 1 and 2 or 3 and 4 in Table 4 . The relative insensitivity of CLL adsorption facility to heating is consistent with the results of Metcalfe et al. (10) who found that heating did not affect the ability of extracted lung lipids to adsorb and subsequently generate low minimum surface tensions in a bubble apparatus. The absence of detrimental heating effects on CLL dispersions is also consistent with mixture fluidity considerations. Phospholipid dispersions above $\mathbf{T}_{c}$ are mobile, and can freely act to minimize their free energy in an aqueous dispersion. For complex, multicomponent mixtures such as CLL it is difficult to define a single meaningful $T_{c}$ value. Nonetheless, this mixture has a high \% of phospholipids with unsaturated fatty chains, and is thus relatively fluid at room temperature. Consequently, addi-

Table 4. Adsorption of CLL and 50:50 DPPC:CLL mixtures

\begin{tabular}{llccccc}
\hline \multicolumn{1}{c}{ Mixture } & $\begin{array}{c}\text { Dispersal } \\
\text { method }^{1}\end{array}$ & $\begin{array}{c}\pi \text { at } \\
1 \mathrm{~min}^{2}\end{array}$ & $\begin{array}{c}\pi \text { at } \\
5 \mathrm{~min}\end{array}$ & $\begin{array}{c}\pi \text { at } \\
10 \mathrm{~min}\end{array}$ & $\begin{array}{c}\pi \text { at } \\
30 \mathrm{~min}\end{array}$ \\
\hline 1. CLL & V/NH/NA & 11 & 23 & 46 & 46 & $(n=3)$ \\
2. CLL & V/H/NA & 19 & 30 & 46 & 47 & $(n=2)$ \\
3. CLL & $\mathrm{S}_{\mathrm{i}} / \mathrm{NH} / \mathrm{NA}$ & 45 & 46 & 47 & 47 & $(n=2)$ \\
4. CLL & $\mathrm{S}_{\mathrm{i}} / \mathrm{H} / \mathrm{NA}$ & 45 & 45 & 46 & 47 & $(n=2)$ \\
5. $50: 50$ & V/NH/NA & 4 & 13 & 22 & 28 & $(n=2)$ \\
$\quad$ CLL: & & & & & & \\
DPPC & & & & & & \\
6. 50:50 & $\mathrm{S}_{\mathrm{i}} / \mathrm{NH} / \mathrm{NA}$ & 37 & 41 & 45 & 46 & $(n=2)$ \\
CLL: & & & & & & \\
DPPC & & & & & & \\
\hline
\end{tabular}

'Total bulk lipid concentration same as Table $1,2,3: 62.5 \mu \mathrm{g} / \mathrm{ml}$.

${ }^{2} \pi$ values are averages of $n$ experiments.

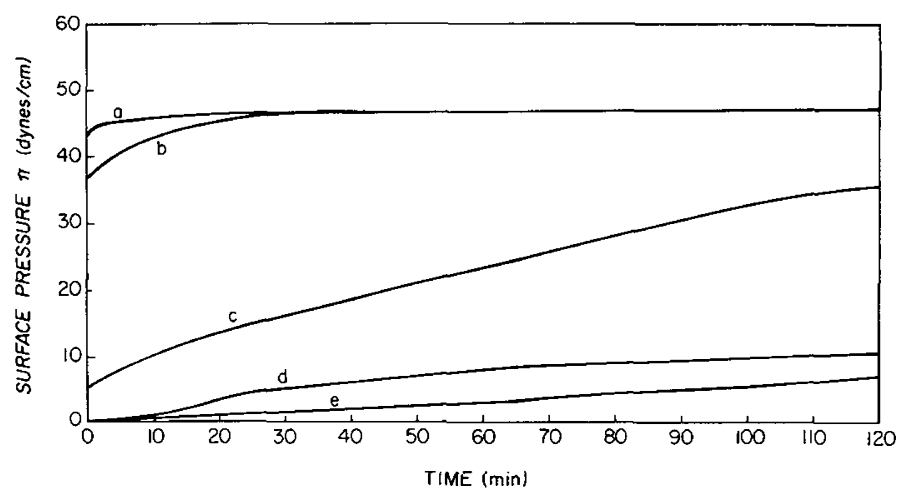

Fig. 2. Adsorption characteristics of different phospholipid mixtures. Cases shown are representative experiments for the case of $S_{i} / N H / N A$, which tended to give maximal or nearly maximal adsorption for the given mixture (but see footnote 24). (a) Mixed lipids (CLL); (b) 5:5 DPPC:CLL; (c) 7:3 DPPC:PG; (d) 9:1 DPPC:DPPE; and (e) DPPC.

tional heating should not significantly change available solution configurations, and hence would not have a large effect on adsorption.

The data of Table 4 show that our CLL adsorbed well on 0.15 $\mathrm{M} \mathrm{NaCl}$, without the necessity for significant protein concentrations. This finding is again in agreement with the recent results of Metcalfe et al. (10). King and Macbeth (8) have suggested that lung surfactant apoprotein might play an important role in the adsorption process, but this does not seem to be supported by our results. Such a role is not completely ruled out, however, because a small protein concentration of about $0.5-0.75 \%$ was found in our CLL preparation.

A final point of interest with regard to the data for CLL is that adsorption of this mixture was affected by the addition of DPPC. Specifically, when DPPC was added to CLL in an approximately equimolar mixture, the adsorption was retarded compared to CLL alone (cases 5 and 6 in Table 4). The degree of retardation was greatest for vortexed mixtures of DPPC:CLL (case 5) as opposed to sonicated mixtures (case 6) but some effect is seen for both preparation procedures. In terms of relevance for lung surfactant replacement, this result suggests that too much DPPC in a replacement mixture might actually be detrimental rather than beneficial. Although the excess DPPC may lead to improved dynamic surface tension lowering in some cases, it might also decrease the rapidity of adsorption. The impact of these competing effects cannot accurately be assessed until it is known what adsorption magnitude and time-scale is critical for replacement therapy.

In summary, we have shown that the adsorption of phospholipid mixtures to the air-water interface can be a function of the path by which the molecules are dispersed in the aqueous bulk phase. This path dependence correlates with the various metastable bulk solution states possible for phospholipid molecules below $T_{c}$. It appears that heating phospholipid dispersions to $\mathrm{T}=45^{\circ} \mathrm{C}$ may inhibit adsorption by allowing the formation of more stable bulk solution states, or by affecting the ease and uniformity of dispersion of mixture components. Methods such as ice sonication, which give rise to increased liposomal surface area compared to vortexed dispersions, were found in general to increase adsorption facility. For example, the fact that 7:3 DPPC:PG adsorbs somewhat more rapidly if dispersed in water by sonication in an ice bath rather than by vortexing suggests that the adsorption process is at least partially dependent on liposomal size for this system. However, additional experiments including the control of possible heating effects in the vortexing process are needed to clarify this interpretation.

In general, the magnitude of path dependent effects in terms of surface tension lowering behavior with time was found to be largest for the case of 7:3 DPPC:PG mixtures (Fig. 1); but some path dependence was also apparent for DPPC, 9:1 DPPC:DPPE, and CLL dispersions. Of the systems studied, mixed lipids ex- 
tracted from calf lung lavage were unique in exhibiting extremely rapid adsorption (in sec) to surface pressures of $46-47$ dynes $/ \mathrm{cm}$, equivalent to surface tensions of $22-23$ dynes $/ \mathrm{cm}$ at $37^{\circ} \mathrm{C}$. These results are apparent on Figure 2 , which shows explicit $\pi$-time behavior for the adsorption of DPPC, 7:3 DPPC:PG, 9:1 DPPC:DPPE, CLL and 5:5 DPPC:CLL. Results shown in Fig. 2 are representative experiments for the dispersion method of $\mathrm{S}_{\mathrm{i}} /$ NH/NA which was found to give maximal or near-maximal adsorption for each system in terms of the preparation methods tested here (24).

The advantageous adsorption properties shown for CLL in Table 4 and on Figure 2 may be related to the success of extracted natural preparations over synthetic preparations in animal model studies of RDS therapy. In addition, the path dependence found in the adsorption of DPPC containing mixtures implies that this surface property should be characterized as a function of dispersion method for each specific phospholipid mixture under evaluation for possible therapeutic use.

\section{REFERENCES AND NOTES}

1. Bligh, E. G. and Dyer, W. J.: A rapid method of total lipid extraction and purification. Can. J. Biochem. Physiol., 37: 911 (1959).

2. Blume, A. and Ackerman, T.: A calorimetric study of the lipid phase transitions in aqueous dispersions of phosphorylcholine-phosphorylethanolamine mixtures. FEBS Letters, 43: 71 (1974)

3. Finkeistein, J, and Weissmann, G.: The introduction of enzymes into cells by means of liposomes. J. Lipid Res., 19: 289 (1978).

4. Gershfeld, N. L.: Equilibrium studies of lecithin-cholesterol interactions: I. Stoichiometry of lecithin-cholesterol complexes in bulk systems. Biophys. J., 22: 469 (1978).

5. Gregoriadis, G.: The carrier potential of liposomes in biology and medicine. N. Engl. J. Med., 295: 704 (1976).

6. Horn, L. W. and Gershfeld, N. L.: Equilibrium and metastable states in lecithin films. Biophys. J., 18: 301 (1977).

7. King, R. J. and Clements, J. A.: Surface active materials from dog lung. I. Methods of isolation, II. Composition and physiological correlations, III. Thermal analysis. Amer. J. Physiol., 223: 707, 715, 727 (1972).

8. King, R. J. and Macbeth, M. C.: Physiochemical properties of dipalmitoyl phosphatidylcholine after interaction with an apolipoprotein of pulmonary surfactant. Biochim. Biophys. Acta, 557: 86 (1979).

9. Lowry, O. H., Rosebrough, N. J., Farr, A. L. and Randall, R. J.: Protein measurement with the folin phenol reagent. J. Biol. Chem., 132: 265 (1951).

10. Metcalfe, I. L., Enhorning, G. and Possmayer, F.: Pulmonary surfactant-associated proteins: their role in the expression of surface activity. J. Appl. Physiol., 49: $34(1980)$.

11. Morley, C., Robertson, B., Lachmann, B., Nilsson, R., and Bangham, A.: Artificial surfactant and natural surfactant: comparative study of the effects on premature rabbit lungs. Arch. Dis. Child, 55: 758 (1980).

12. Morley, C. J., Miller, N., Bangham, A. D. and Davis, J. A.: Dry artificial lung surfactant and its effect on very premature babies, The Lancet, 1: 64 (1981).

13. Notter, R. H. and Morrow, P. E.: Pulmonary surfactant: a surface chemistry viewpoint. Ann. Biomed. Engr., 3: 119 (1975).

14. Notter, R. H. and Shapiro, D. L.: Lung Surfactant in an era of replacement therapy. Pediatrics, 68: 781 (1981).

15. Phillips, M. C. and Hauser, H.: Spreading of solid glycerides and phospholipids at the air-water interface. J. Colloid Interface Sci., 49: 31 (1974).

16. Robertson, B.: Surfactant substitution: experimental models and clinical applications. Lung, 158: 57 (1980)

17. Robertson, B.: Neonatal pulmonary mechanics and morphology after experimental therapeutic regimens. In: Scarpelli, E. M. (Ed) Reviews in Perinatal Medicine, Volume 4, p. 337, (Raven Press, New York, 1981).

18. Shimshick, E. J. and McConnell, H. M.: Lateral phase separation in phospholipid membranes. Biochemistry, 12: 2351 (1973).

19. Tajima, K. and Gershfeld, N. L.: Detection of low levels of lipid contamination in lecithin by equilibrium spreading pressures. J. Colloid Interface Sci., 81: 283 (1981)

20. Touchstone, J. C., Chen, J. C. and Beaver, K. M.: Improved separation of phospholipids in thin layer chromatography. Lipids, 15:61 (1980).

21. Villalonga, $F$.: Surface chemistry of $\mathrm{L}-\alpha$-dipalmitoyl lecithin at the air-water interface. Biochim. Biophys. Acta., 163: 290 (1968).

22. In all cases, a uniform time period of $1 \mathrm{~min}$ was used for sonication at a low power setting of 25 watts. Similarly, a uniform total time interval of $1 \mathrm{~h}$ was used for all vortexed dispersions. Because the ease of phospholipid dispersion varied as a function of mixture composition, this time standardization meant that in some cases all $5 \mathrm{mg}$ of surface active material were not uniformly dispersed in solution. In experiments with pure DPPC, particles of phospholipid were sometimes visible on the side of the mixture container after vortexing (or sonication to a lesser extent) was completed. Thus, the amount of phospholipid completely dispersed in solution was actually less than $5 \mathrm{mg} / \mathrm{ml}$ in some experiments, depending upon the efficacy of a given dispersion method. However, it was felt that comparisons of adsorption facility between different phospholipid mixtures would be less subjective if the various dispersion methods were standardized in terms of time for all of the mixtures tested.

23. The characteristics of the initial phase of adsorption for vortexed CLL dispersions are composition dependent. For a different CLL preparation than used in Table 4, we found a more rapid initial adsorption phase after vortexing, with a less prominent point of inflection in initial $\pi$ - $t$ behavior. These differences emphasize the importance of characterizing adsorption behavior explicitly for extracted lung surfactant mixtures as well as for synthetic mixtures.

24. Our results are not intended to define a precise protocol necessary to yield maximal adsorption for a specific surface active preparation. Other dispersion methodology than used here may give increased adsorption for a specific mixture. Similarly, increased bulk concentration might lead to increased adsorption, especially for some of the synthetic phospholipid mixtures. The methods studied here were primarily chosen to display the existence of path dependent behavior in the phospholipid adsorption process.

25. Requests for reprints should be addressed to: Dr. Robert H. Notter, University of Rochester Medical Center, Department of Pediatrics, 601 Elmwood Ave., Box 777, Rochester, NY 14642.

26. This research was supported by NIH Grant HL-25170 and by RCDA 1K04-HL00945. S.S. was supported by a work/study program from Xerox Corporation. 27. Received for publication July 16,1981 .

28. Accepted for publication November 23, 1981. 